This document is the accepted manuscript version of a published work that appeared in final form in Journal of physical chemistry $C$, copyright (c) American Chemical Society after peer review and technical editing by the publisher. To access the final edited and published work see

DOI 10.1021/acs.jpcc.5b01263

The posting must be for non-commercial purposes and not violate the ACS' "Ethical Guide.

This version is published under a "All rights reserved" license. 


\title{
High Aspect Ratio Gold Nanorods grown with Platinum Seeds
}

\author{
Miriam Varón, a Jordi Arbiol, ${ }^{\text {b,c }}$ and Víctor F. Puntes, ${ }^{\text {a,c,* }}$ \\ ${ }^{a}$ Catalan Institute of Nanotechnology (ICN2) and Universitat Autònoma de Barcelona \\ (UAB), Campus de la UAB, 08193 Bellaterra (Barcelona) \\ ${ }^{b}$ Institut de Ciència de Materials de Barcelona (ICMAB-CSIC),Campus UAB, 08193 \\ Bellaterra, Spain

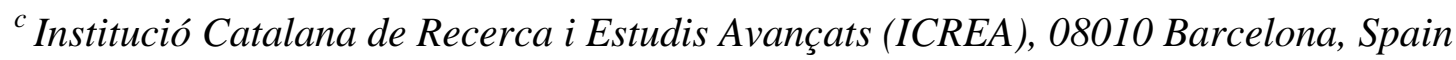 \\ *Corresponding author: E-mail address: victor.puntes@icn.cat
}

\begin{abstract}
Using Au chloride as precursor, Pt nanocrystals as seeds, ascorbic acid (AA) as a reducer and hexadecyltrimethylammonium bromide, (CTAB) as surfactant and complexing agent, extremely long Au nanorods (NRs) have been grown. The influence of different parameters such as the composition of the seed particles, the concentration of Pt precursor or the type of Pt source present in solution have been analyzed. These large Au NRs have been exhaustively characterized by advanced electron microscopies, (S)TEM, SEM, HR-TEM and optical microscopy, as well as UV-Vis spectroscopy and their morphology correlated with the growth mechanism.
\end{abstract}

Keywords: Au nanocrystals, high aspect ratio nanorods, crystal growth, 


\section{Introduction}

In the last years, to harness properties of the materials at the nanometer scale, size and shape control has been, and still is, one of the most important issues in nanotechnology. Consequently, a wide range of synthetic methods have been reported in the literature describing the preparation of nanoparticles (NPs) with different morphologies. ${ }^{1}$ A special case are long nanorods (NRs) (also called nanowires (NWs)) which are specially interesting due to their capacity to be integrated into modern nanocircuitry and flexible electronics, isothermal fabrics, plasmonic propagation devices ${ }^{2}$ and plasmonic antennas, ${ }^{3}$ and their emerging use to wire regenerative tissue ${ }^{4,5}$, among many other benefits resultant from their very high anisotropy, as their special (toxic) biological effects related to frustrated phagocytosis. ${ }^{6}$ Different chemical approaches have been attempted to produce long gold NRs (Au NRs) by reduction methods in aqueous surfactant media such as electrochemical, ${ }^{7}$ photochemical ${ }^{8}$, or seed-mediated growth approaches. ${ }^{9}$ Initially, pre-synthesized small particles (seeds) were used as nuclei for the anisotropic growth of NRs as far as $30-40 \mathrm{~nm}$ in width and hundreds of nanometers in length. ${ }^{10,11}$ In those cases, the particle size was controlled by varying the ratio between metal salt and (citrate-stabilized) Au seeds, while their aspect ratio (AR) was increased by increasing $\mathrm{Ag}^{+}$ion concentration (AR between 2 and 18). ${ }^{10}$ El-Sayed and co-workers reported improvements of the Au seed-mediated growth method by replacing citrate by hexadecyltrimethylammonium bromide (CTAB) stabilizing molecules, which resulted in the formation of single crystalline cylindrical Au NRs with ARs below 10 in very high yields. ${ }^{12}$ Later, the addition of an appropriate amount of nitric acid to the growth solution produced Au NRs with high AR (> 20) in high yield, ${ }^{13,} 14$ that were further grown up to ARs of $200(10 \mu \mathrm{m}, 50 \mathrm{~nm})$ by decreasing the initial amount of seed particles. ${ }^{15}$ The role of the $\mathrm{pH}$ has been also recently studied in detail leading to the 
adjustment of NRs length (between 1 to $6 \mu \mathrm{m}$ ) modifying the $\mathrm{pH}$ of the solution. ${ }^{16}$ At these large sizes, in the growth conditions, NRs started being colloidally unstable and sediment, resulting in phase segregation, where gelation can occur ${ }^{13}$. In these conditions, the growth process is impaired, therefore limiting the further NRs growth. Besides, the benefit of using Pt as highly catalytic seeds for the growth of Ag NWs has been recently reported ${ }^{17}$. Basically, from the synthetic point of view, the control of NR dimensions relies on the independent control of the NC nucleation and growth (i.e. the balance between the preparation of many short rods or few very long rods), a challenging situation to perform in just one step; therefore, multistep seeded growth methods need to be developed to grow large NCs. This is because most of actual wet chemistry methods for NP production are based on the Lamer supersaturation approach. ${ }^{18}$ In this process, the sudden monomer supersaturation provided by highly unstable precursors leads to a burst nucleation and a rapid growth, depleting monomer from the solution and favoring monodispersity ${ }^{19}$. In these conditions, the size of the resultant particles is limited due to the fact that at certain point, the addition of more precursor leads to more, but not larger, NRs.

In the present work, we report the synthesis of extremely long NRs using Pt NCs as catalytic seeds for the reduction of a less active Au precursor. The decrease of precursor reactivity was achieved by the use of surfactants at high concentrations and taking advantage that the energy barrier for nucleation is higher than for growing. As a result, the slowly growth of Au NRs for days (30 days) is obtained, since there is enough raw material to grow very large crystals but always present at low concentrations to avoid new nucleation. These large Au NRs have been exhaustively characterized by (S)TEM, SEM and optical microscopy as well as UV-Vis spectroscopy and their bending morphology correlated with the growth mechanism. 


\section{Experimental Section}

Chemicals. Hydrogen tetrachloroaurate trihydrate $\left(\mathrm{HAuCl}_{4} \cdot 3 \mathrm{H}_{2} \mathrm{O}\right)$, potassium tetrachloroplatinate(II) $\left(\mathrm{K}_{2} \mathrm{PtCl}_{4}\right)$, hexadecyltrimethylammonium bromide (CTAB) 95\%, trisodium citrate $99 \%$, sodium borohydride $\left(\mathrm{NaBH}_{4}\right)$ and ascorbic acid (AA) were purchased from Sigma-Aldrich and used as received without further purification.

Synthesis of CTAB stabilized Pt NPs seeds. The synthesis of $\sim 3$ nm Pt NCs stabilized in CTAB was carried out following the method described by Grzelczak et al. ${ }^{20} \mathrm{~A}$ mixture of $9.63 \mathrm{~mL}$ of CTAB $0.1 \mathrm{M}$ with $50 \mu \mathrm{L}$ of $\mathrm{K}_{2} \mathrm{PtCl}_{4} 0.05 \mathrm{M}$ was heated up to 40 ${ }^{\circ} \mathrm{C}$ for $5 \mathrm{~min}$ until the solution became clear. After adding $0.3 \mathrm{~mL}$ of $\mathrm{NaBH}_{4} 0.06 \mathrm{M}$, the vial was capped immediately. After $10 \mathrm{~min}$, the vial was opened and stirred for several minutes until the decomposition of the $\mathrm{NaBH}_{4}$. NP concentration was of $\sim 5 \cdot 10^{14}$ $\mathrm{NP} / \mathrm{mL}$, in agreement with those previously reported for Au seeds. ${ }^{21}$

Synthesis of CTAB stabilized Au NPs seeds. The synthesis of Au NPs stabilized in CTAB were prepared via the method developed by Nikoobakht and El-Sayed. ${ }^{12}$ Briefly, $5 \mathrm{~mL}$ CTAB solution $0.2 \mathrm{M}$ was mixed with $5 \mathrm{~mL}$ of $\mathrm{HAuCl}_{4}$ solution $0.0005 \mathrm{M}$. Next, 0.6 $\mathrm{mL}$ of freshly prepared $\mathrm{NaBH}_{4} 0.01 \mathrm{M}$ was added to the mixture while vigorous stirring, which resulted in the formation of a light-brown solution within few seconds. Stirring of the solution was continued for 2 min.

Synthesis of Au NRs with Pt seeds. Growth solution was prepared by mixing $5 \mathrm{~mL}$ of CTAB $0.2 \mathrm{M}$ and $5 \mathrm{~mL}$ of $\mathrm{HAuCl}_{4} 0.001 \mathrm{M}$. After that, $50 \mu \mathrm{L}$ of AA $0.1 \mathrm{M}$ was added as a mild reducing agent, changing the growth solution from dark yellow to colorless. Then, a volume between 5 and $30 \mu \mathrm{L}$ of the pre-synthesized Pt seeds was added and 
gently mixed for 10 seconds. The solution was kept at RT without stirring for 1 month. The evolution of the reaction was followed by UV-vis, ICP-MS and TEM.

Synthesis of Au NRs with Au seeds. Three different volumes (0.015 mL, $0.15 \mathrm{~mL}$ and $1.5 \mathrm{~mL}$ ) of $\mathrm{K}_{2} \mathrm{PtCl}_{4}$ solution $0.004 \mathrm{M}$ were added to $5 \mathrm{~mL}$ CTAB solution $0.2 \mathrm{M}$. Then, $5 \mathrm{~mL}$ of $\mathrm{HAuCl}_{4} 0.001 \mathrm{M}$ and $55 \mu \mathrm{L}$ of AA $0.1 \mathrm{M}$ were added simultaneously to the solutions dropwise, and then gently mixed for 10 seconds. It is worth noting that the three solutions above are identical except for the Pt ion content. The final step was the addition of $12 \mu \mathrm{L}$ of the pre-synthesized Au NP seed solution to the growth solutions. The solutions were kept at RT.

Characterization. Au NRs were characterized by optical microscope, scanning electron microscopy (SEM), transmission electron microscopy (TEM) and High resolution TEM (HRTEM), and UV-vis spectroscopy. TEM analysis was performed on a JEOL 1010 with an accelerating voltage of $80 \mathrm{kV}$ and a digitalization image system Bioscan (Gatan). HRTEM was performed on a JEOL JEM 2010F field emission gun microscope with an accelerating voltage of $200 \mathrm{kV}$. For the Z-contrast imaging we used a high angle annular dark field (HAADF) detector coupled to the same microscope, which allows working in scanning TEM (STEM) mode. The as-synthesized Au NR solutions were concentrated and separated from the small nanospheres and surfactant by centrifugation. A volume of $1 \mathrm{~mL}$ of solution was centrifuged at $1500 \mathrm{rpm}$ for $20 \mathrm{~min}$. The supernatant was removed with a syringe and the precipitate was redispersed in 20 $\mu \mathrm{L}$ of distilled water. Finally, the concentrated solution was dropped onto a carbon coated copper grid. SEM analysis was performed on a MERLIN Field Emission SEM (Fe-SEM) from Zeiss with an operating voltage of $1.2 \mathrm{kV}$. The Au NR solution was 
dropped on a highly ordered pyrolytic graphite (HOPG) substrate, and after 5 min the excess was removed tilting the substrate onto an absorbent paper. Then was rinsed with methanol and distilled water to remove CTAB. For optical images, Axio Observer z1m from Zeiss was used. The sample deposition onto a mica substrate was prepared by means the same procedure explained above. UV-vis spectra were obtained with a Shimadzu UV-2401PC spectrophotometer, over the range of 200-1000 nm with a $1 \mathrm{~cm}$ path length quartz cuvette after sample re-suspension to avoid exclusion of large crystals that slowly sediment.

\section{Results}

In this work we present a simple and reproducible method for preparing high AR Au NRs (as $20 \mu \mathrm{m}$ length and $36 \mathrm{~nm}$ in diameter, $500 \mathrm{AR}$ ), to our knowledge the largest one reported up to date. Note that hundred thousand of $10 \mathrm{~nm}$ Au spherical NPs $\left(\sim 10^{4}\right.$ atoms/NP) fit into one single Au NR (( $10^{9}$ atoms/NP), therefore, large amounts of Au precursor are required, which normally causes additional nucleation and increased fraction of byproducts. To overcome that, the chemical potential of the reaction was decreased, basically by increasing the concentration of complexing agents, lowering the reaction temperature and passivating NPs surface, thus new nucleation is avoided while the NRs grow up to very large sizes. The reaction has been performed in a closed system let for one month at RT without any stirring (Figure 1). These conditions should enable easy scaling up of the production of NRs thanks to the absence of temperature gradients and small mass gradients during synthesis. The use of Pt is thus justified as a reduction catalyst, non-miscible with Au at RT and not so easily oxidized by Au cations in the reaction mixture, favoring growth. 


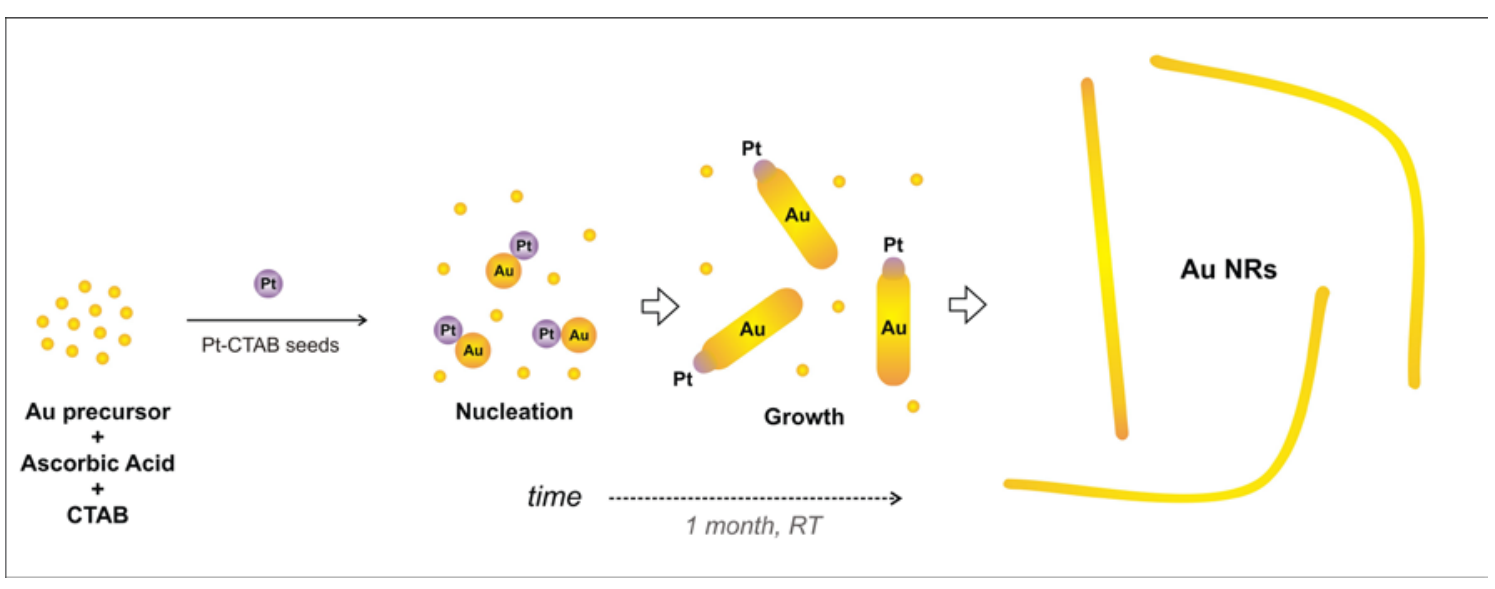

Figure 1. Schematic representation of the seeding-growth method for the synthesis of long Au NRs (drawn not at scale).

Thus, following the procedures reported in the experimental section, high AR Au NRs have been obtained. Figure 2 shows TEM images of as-synthesized rods after 1 day (A), 1 week (B), 2 weeks (C) and 1 month (D) in the growth solution, employing CTAB-capped Pt seeds. From the size distribution diagram after 1 day reaction (Figure 2A, inset), it can be observed that the Au NRs have lengths between 100 and $600 \mathrm{~nm}$ and a mean width of $26 \pm 5 \mathrm{~nm}$. The average length increases to $\sim 1 \mu \mathrm{m}$ at 1 week, and at 15 days it is of few microns (average $\sim 5 \mu \mathrm{m}$ ). After 1 month in the reaction mixture, Au NRs have grown until reaching lengths of several microns ( $>10 \mu \mathrm{m})$ and a mean width of $36 \pm 11 \mathrm{~nm}$ (see more TEM images of Au NRs in Figure S1 of the supporting information). The synthesis of NRs is always accompanied by the formation of byproducts, spherical particles and plates. This is the highest growth rate and concentration we can achieve before producing too much bystanders (no rod-shaped NPs). Figure 2E shows the normalized UV-vis absorption spectra of the growth solution at different times. It can be seen that by increasing the reaction time the peak broadens and new features appear at higher wavelengths, as the transverse plasmon 

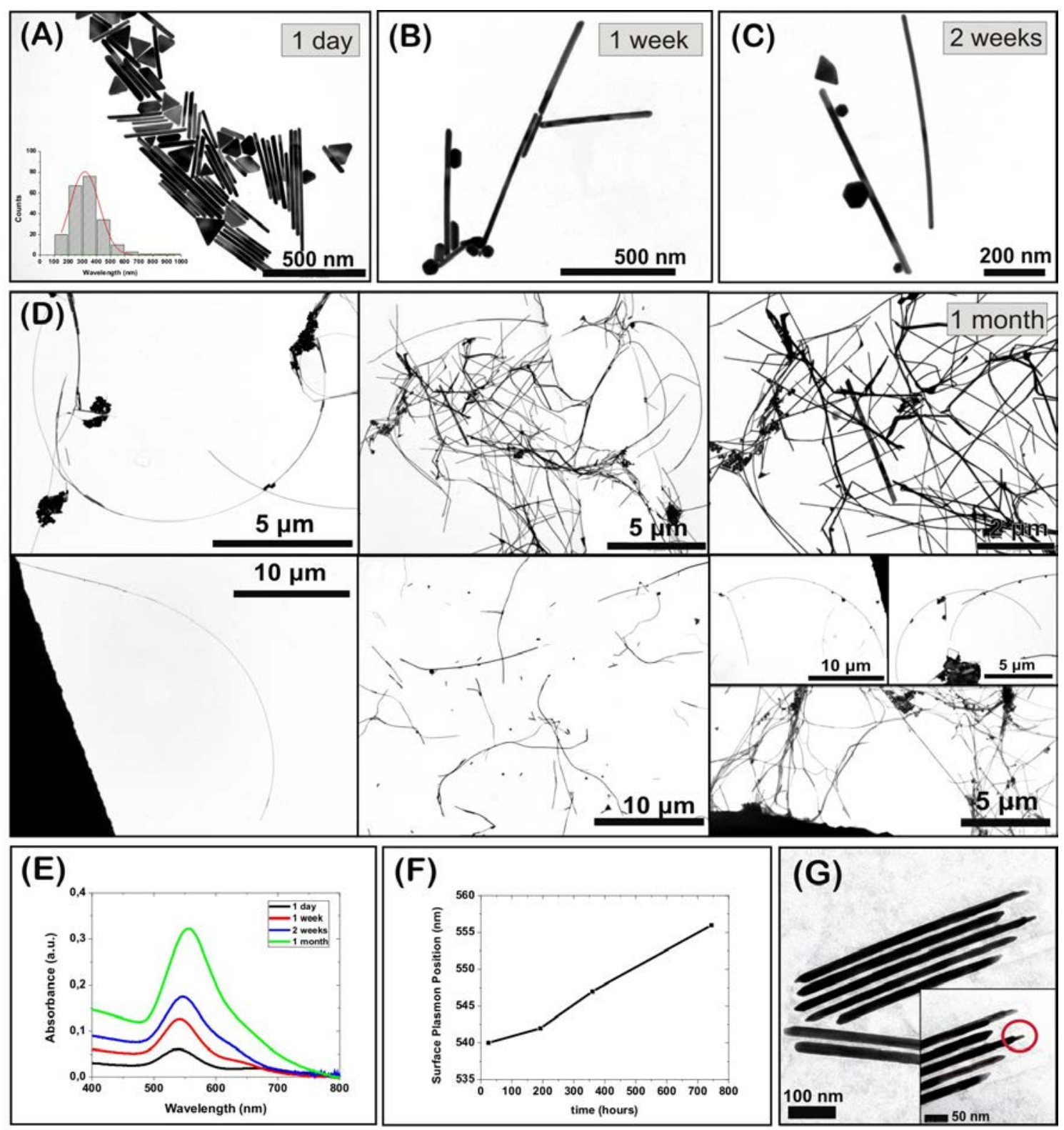

Figure 2. TEM images of the Au NRs produced by a seeding growth method in presence of metallic Pt at different times: A) 1 day (inset: size distribution diagram of the length of Au NRs after 1 day reaction), B) 1 week, C) 2 weeks and D) 1 month. E) Normalized $U V$-visible spectrum of the growth of the Au NR solution right after addition of $30 \mu L$ of Pt seeds. F) Variation of the longitudinal surface plasmon peak with time. Only the transverse plasmon band of Au NRs is observed since the longitudinal peak appears al higher wavelengths at the IR range. The length of the NRs increases up to a certain limit after 1 month when no longer growth is observed. G) TEM image of Au NRs after 1 day reaction, where it can be observed an elongated tip at the end of the NRs. 


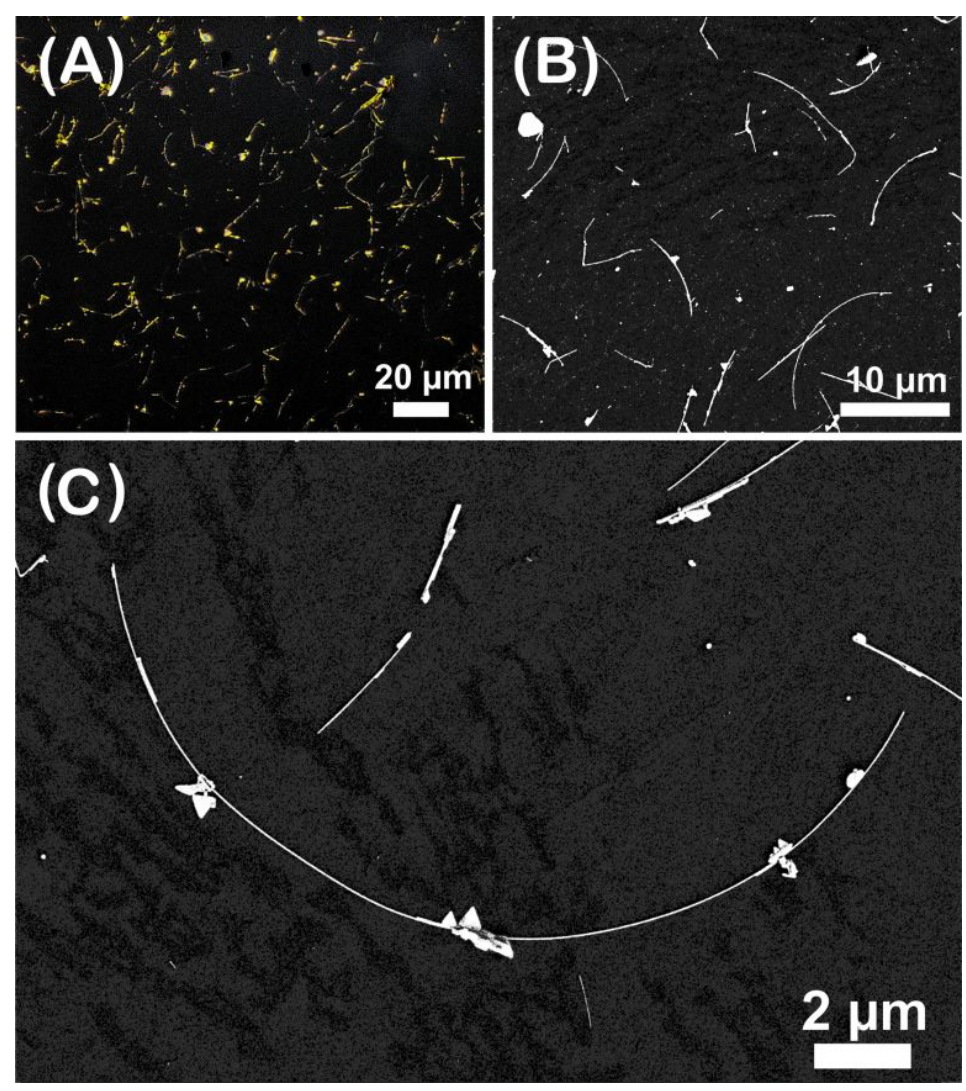

Figure 3. Optical microscope image of the extremely Au NRs deposited onto a mica substrate (A) and SEM images of the Au NRs up to $20 \mu \mathrm{m}$ deposited onto a HOPG substrate (B and C). 
To investigate if the growth of Au NRs after 1 month was indeed still due to the $\mathrm{Au}^{+3}$ precursor present in the growth solution or by the dissolution of other Au NPs (Ostwald ripening), the Au-content of the reaction mixture was monitored by ICP-MS. Samples at different time intervals were destabilized by adding organic solvents and fractioned by centrifugation and the precipitates and supernatant were analyzed. Analysis showed a 46 \% Au consumption after 1 day, $53 \%$ after 1 week and $84 \%$ after 1 month. Similarly, results were obtained when double amount of reagents were used, observing a similar trend (38\% after 1 day, $40 \%$ after 1 week and $81 \%$ after 1 month). No presence of elemental Pt was detected on the supernatant by ICP-MS in any case while it was detected in the pellet. Interestingly, at intermediate times, morphological characterization revealed the presence of a narrow tip at the end of the rods (Figure 2G). This observation recalls, among others, to the colloidal synthesis of Ge NRs reported by Korgel group. ${ }^{25,26}$. Even more significant, in control experiments, no NR growth was observed by using Au or Ag seeds in the same conditions, likely due to the increased inertness of $\mathrm{Au}$ and the lower redox potential of Ag. Note that Pt and Au are immiscible at RT and that therefore, at some point, there should be a Pt domain in the grown rods. Unfortunately, the presence of Pt cannot be confirmed neither by EDX nor EELS which can be explained by the strong signal overlapping of Au and Pt in EDX, and by the poor and also overlapped signal of Au and Pt in EELS (Figure 4), due to their proximity in the periodic table. However, the absence of Pt in the supernatant and its presence in the precipitate after centrifugation (observed by ICP-MS) indicates that Pt is incorporated in the growing rod. Other control studies were performed to further understand the role of the Pt seeds in the AuNR formation. First, in the absence of metallic seeds, no formation of Au particles was observed in the growing mixture at RT, indicating that the $\mathrm{AA}$ is too weak to reduce $\mathrm{Au}^{+}$in the presence of CTAB in these 
conditions. Second, by modifying the amount of seeds, we observed that at low concentrations or in the absence of Pt seeds (replaced by Au seeds) resulted into less byproduct formation but shorter rods (Figure 5 A-D). Besides, a higher concentration of Pt-seeds resulted in a higher presence of byproducts that led to a redshift of the transverse plasmon band, indicative of both, an increase of the NRs width as well as an increase in the number of by-products in solution. Finally, to study if the ionic form of platinum $\left(\mathrm{Pt}^{+2}\right)$ had some effect in the growth process, we performed similar experiments but replacing the Pt-seeds by equivalent amounts of the $\mathrm{K}_{2} \mathrm{PtCl}_{4}$ salt. In this set of experiments, we also employed Au-seeds synthesized in CTAB to catalyze the reaction. As it can be observed in Figures $5 \mathbf{F}-\mathbf{H}$, when the amount of $\mathrm{Pt}^{+2}$ was increased, the rod size decreased gradually until polydisperse spherical Au NPs of about $200 \mathrm{~nm}$ were finally formed. We can conclude that, under the reported conditions, the presence of low concentrations of metallic Pt facilitates the growth of large Au NRs, while the presence of ionic $\mathrm{Pt}^{+2}$ hampers it. Finally, we also note that the mixture of CTAB, AA and $\mathrm{Pt}^{+2}$ is not enough to produce Pt NPs at RT even after one month of incubation. 


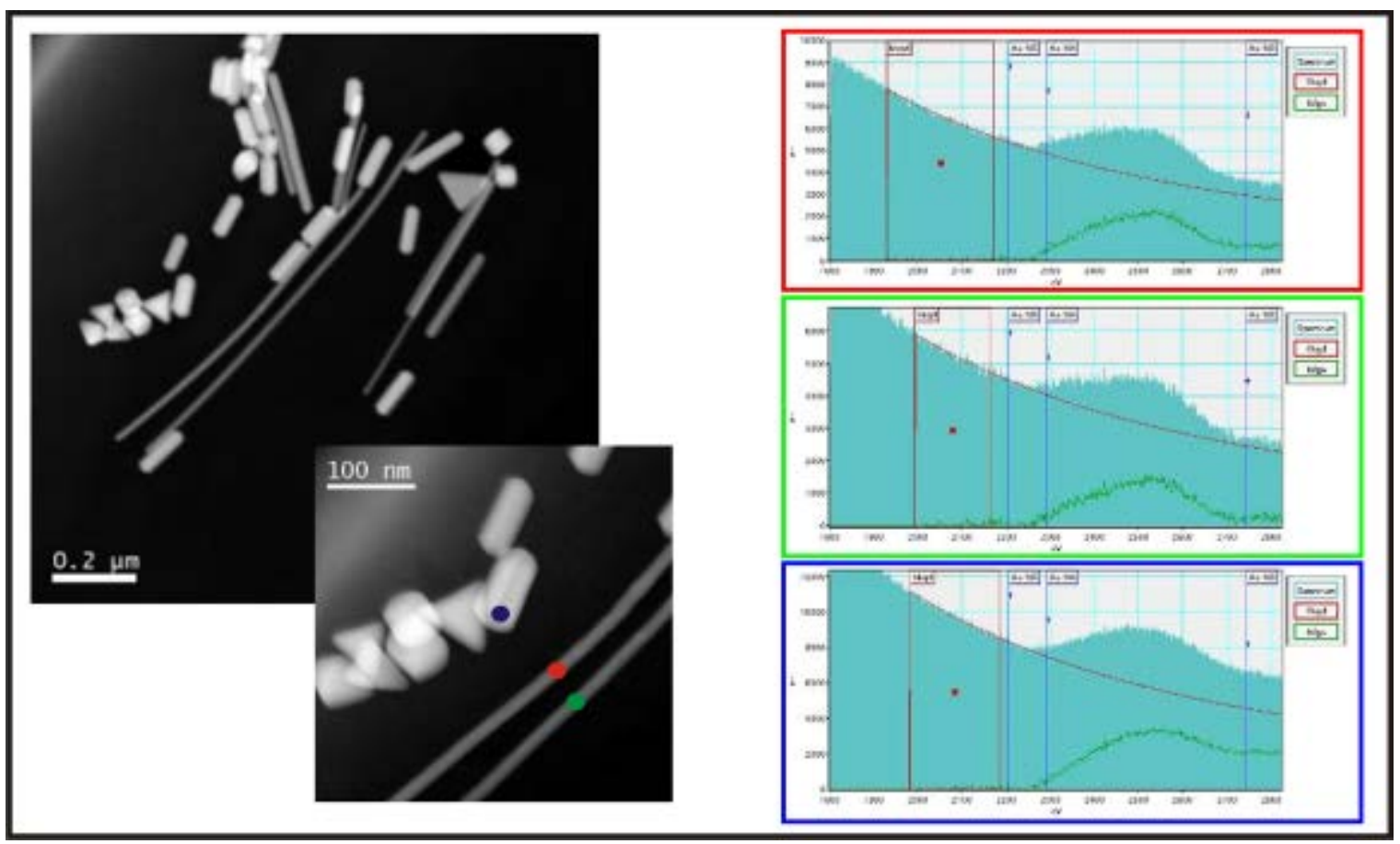

Figure 4. Z-contrast image and EELS spectra of three different zones. Every color mark in the Z-contrast image corresponds to the respective color frame EELS analysis. The EELS analyses show that both the particles and the long NRs are formed by gold. No signal of Pt was found, but is remarkably to mention that both signals of gold and Pt overlap in the EELS spectra.

The presence of the surfactant is also critical in the formation process. It controls the kinetics of the reaction by interacting with the growing NCs, ions and intermediates present in solution ${ }^{27-29}$, in addition to passivate the NC surface against aggregation and to promote anisotropic or faceted growth ${ }^{30}$. It is known that the AuNR growth rate in water is controlled by the collisions of the $\mathrm{Au}^{+}-\mathrm{CTAB}$ metallo-micelles with the formed rods. ${ }^{31}$ In fact, a high concentration of CTAB (up to $0.1 \mathrm{M}$ ) is usually required for NR growth and favour the solubility of Au-CTAB complexes, ${ }^{32}$ even though the critical micelle concentration (CMC) of CTAB is $0.9 \mathrm{mM}$ at RT (also affected by the presence of ions in solution). 

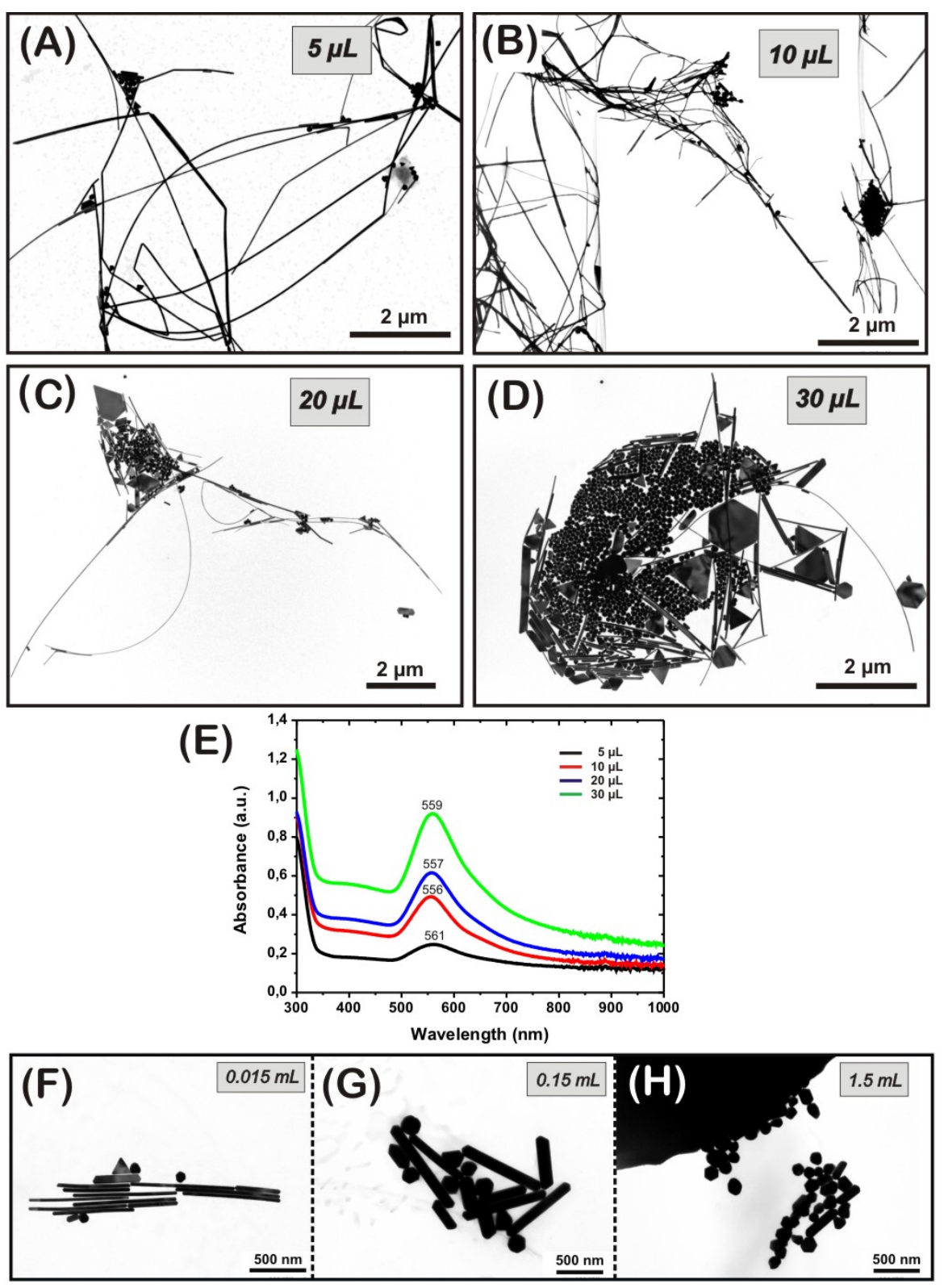

Figure 5. The effect of Pt in the synthetic process. (A-D) TEM images of the synthesis in the presence of different volumes of Pt-seed $\left(\sim 5 \cdot 10^{14} \mathrm{NP} / \mathrm{mL}\right): 5 \mu L(A), 10 \mu L(B), 20$ $\mu L(C)$ and $30 \mu L(D)$. (E) Normalized UV-visible spectrum of the Au NR solution after addition of different volumes of Pt-seeds (5, 10, 20 and $30 \mu L)$ measured after 1 month. (F-H) TEM images showing the $\mathrm{Pt}^{+2}$-salt $\left(\mathrm{K}_{2} \mathrm{PtCl} \mathrm{l}_{4}\right)$ effect in the synthetic process. Different volumes of $\mathrm{K}_{2} \mathrm{PtCl}_{4}$ solution (0.004 M) added: $0.015 \mathrm{~mL}(\mathrm{~F}), 0.15 \mathrm{~mL}(\mathrm{G})$ and $1.5 \mathrm{~mL}(H)$. 
Electrical, magnetic and optical properties of NCs are strongly dependent on atomic structure, defects, grain boundaries and growth orientations, for example, the presence of twins have been shown recently to have a finest effect by changing the local stacking of the atomic planes and thus creating different structural polytypes with different tunable optical and electrical properties. ${ }^{33-37}$ Figure 6 shows HR-TEM images of the Au NRs. The Au NRs, as expected, perfectly crystallize in the Au fcc cubic structure, which is in agreement with previous studies ${ }^{12}$. Remarkably, the grand majority of all the analyzed NRs are single crystals with different number of defects (see more HR-TEM characterization of Au NRs in Figure S2 of the supporting information) suggesting an atom by atom growth. The growth direction of these rods is always along the [1-10] axis. However, the NRs contain plenty of crystalline $\{111\}$ twin and/or stacking fault defects parallel to the growth axis. In both cases, twin defects crossing the entire length of the NR can be observed. These twin defects occur as rotation along the $\{111\}$ planes parallel to the growth axis and tend to occur on the central part of the NR. The HRTEM images are accompanied by the FFT analysis of two different zones, the central and lateral side of a NR, in which the presence of multiple spots on the (-1-11) axis can be found on the power spectrum, thus agreeing with the fact that the twins are partially ordered. In fact, as indicated with the arrows on the inset of Figure 6A, these twins may occur every 2 or $3(-1-11)$ atomic planes, thus forming a kind of ordered twinned superstructure, which can explain the curvature observed in the long Au NRs (crystal strain induces bending). This presence of $\{111\}$ twins parallel to the zone axis is a common feature found in NRs composed of cubic crystalline materials ${ }^{33}$, 36 . It can be also observed in Figure 6B that some of the NRs suddenly change their direction forming an elbow. A further analysis on the bending region confirmed that the shoulder 
is composed of a grain boundary, suggesting a merging area between two different NRs that have fused in some way. During growth, reactive surfaces may easily merge while they are incorporating atoms in a process called pasting or cementation (sintering at low $\mathrm{T}$ while growing). ${ }^{38}$ This grain boundary is not net, meaning that there was not a clear atomic continuity between both sides. In fact, both are just fusing together and creating a boundary. Moirée fringes can be appreciated on the boundary region demonstrating the overlapping of both crystals. In both cases, these boundaries are formed by the fusion of two independent NRs, both growing along the [1-10] axis.

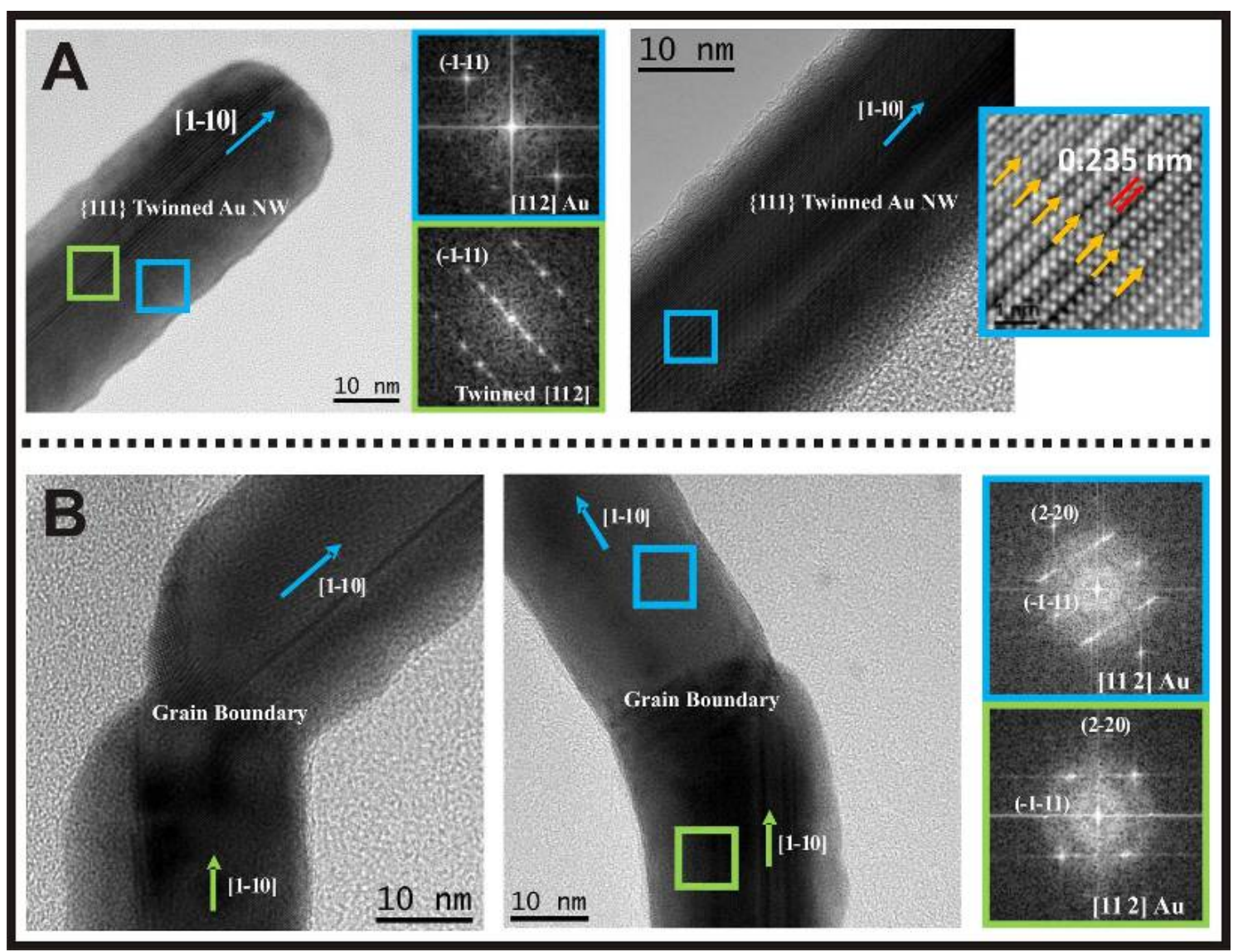

Figure 6. A: panel of HRTEM images of the top part (growth front) of a twinned Au NR (left), with the corresponding FFT patterns of the selected areas, and of a middle section of the same NR (right), showing the twin ordered superstructure in the inset image. B: panel of different grain boundaries between NRs with the corresponding FFT patterns of the selected areas. 


\section{Discussion}

Morphological control is achieved by selective attachment and kinetic/diffusion control $^{24,30,39}$. In here, using Pt seeds at RT, the presence of weak reducers and large amounts of surfactants allows interesting conditions for designed NC growth. ${ }^{40}$ Note that the same process at high temperatures yields multiple Au shapes and very short rods. It is true that the frequently used sequential version -step by step- of the seeded growth process have provided a handy strategy to overgrow NCs, however, limitations exists and careful and costly control of the growth environment is necessary. ${ }^{24}$ The idea here was to prepare a solution that provides reactive monomer to the growing rod at appropriate rates, therefore, avoiding increase of monomer concentration and new nucleation. The production of these long Au rods is promoted by the presence of the Pt seeds, which are incorporated in the rods and probably keep the NR tip "hot” promoting growth in the reacting mixture. Besides, it has also emerged an increasing interest in finding greener methods for NP synthesis. ${ }^{41}$ These green methodologies try to solve problems such as energy consumption (temperature, stirring), the use of toxic reagents and generation of toxic by-products, and scaling-up feasibility, what requires selecting appropriately surfactants, reducers and complexing agents. ${ }^{42}$

Acknowledgements. We acknowledge financial support from Spanish MICINN MAT2006-13572-C02-02-02. MV thanks the Spanish MICINN for the financial support through the fellowship BES-2007-17164, associated to the project. 
Supporting Information Available. Additional TEM images of Au NRs, HRTEM characterization and Z-contrast images. This material is available free of charge via the Internet at http://pubs.acs.org. 


\section{References}

1. Burda, C.; Chen, X.; Narayanan, R.; El-Sayed, M. A., Chemistry and Properties of Nanocrystals of Different Shapes. Chemical Reviews 2005, 105, (4), 1025-1102.

2. Wild, B.; Cao, L.; Sun, Y.; Khanal, B. P.; Zubarev, E. R.; Gray, S. K.; Scherer, N. F.; Pelton, M., Propagation Lengths and Group Velocities of Plasmons in Chemically Synthesized Gold and Silver Nanowires. ACS Nano 2011, 6, (1), 472-482.

3. Paolo, B.; Jer-Shing, H.; Bert, H., Nanoantennas for visible and infrared radiation. Reports on Progress in Physics 2012, 75, (2), 024402.

4. Dvir, T.; Timko, B. P.; Brigham, M. D.; Naik, S. R.; Karajanagi, S. S.; Levy, O.; Jin, H.; Parker, K. K.; Langer, R.; Kohane, D. S., Nanowired three-dimensional cardiac patches. Nat Nanotechnol 2011, 6, (11), 720-5.

5. $\quad$ Tian, B.; Liu, J.; Dvir, T.; Jin, L.; Tsui, J. H.; Qing, Q.; Suo, Z.; Langer, R.; Kohane, D. S.; Lieber, C. M., Macroporous nanowire nanoelectronic scaffolds for synthetic tissues. Nature materials 2012, 11, (11), 986-994.

6. Stoehr, L.; Gonzalez, E.; Stampfl, A.; Casals, E.; Duschl, A.; Puntes, V.; Oostingh, G., Shape matters: effects of silver nanospheres and wires on human alveolar epithelial cells. Particle and Fibre Toxicology 2011, 8, (1), 36.

7. Yu, Y. Y.; Chang, S. S.; Lee, C. L.; Wang, C. R. C., Gold nanorods: Electrochemical synthesis and optical properties. Journal of Physical Chemistry B 1997, 101, (34), 6661-6664.

8. $\quad$ Esumi, K.; Matsuhisa, K.; Torigoe, K., Preparation of Rodlike Gold Particles by Uv Irradiation Using Cationic Micelles as a Template. Langmuir 1995, 11, (9), 32853287.

9. Jana, N. R.; Gearheart, L.; Murphy, C. J., Wet chemical synthesis of high aspect ratio cylindrical gold nanorods. Journal of Physical Chemistry B 2001, 105, (19), 40654067.

10. Jana, N. R.; Gearheart, L.; Murphy, C. J., Seed-Mediated Growth Approach for Shape-Controlled Synthesis of Spheroidal and Rod-like Gold Nanoparticles Using a Surfactant Template. Advanced Materials 2001, 13, (18), 1389-1393.

11. Busbee, B. D.; Obare, S. O.; Murphy, C. J., An Improved Synthesis of HighAspect-Ratio Gold Nanorods. Advanced Materials 2003, 15, (5), 414-416.

12. Nikoobakht, B.; El-Sayed, M. A., Preparation and Growth Mechanism of Gold Nanorods (NRs) Using Seed-Mediated Growth Method. Chemistry of Materials 2003, 15, (10), 1957-1962.

13. Wu, H. Y.; Huang, W. L.; Huang, M. H., Direct high-yield synthesis of high aspect ratio gold nanorods. Crystal Growth \& Design 2007, 7, (4), 831-835.

14. Wu, H. Y.; Chu, H. C.; Kuo, T. J.; Kuo, C. L.; Huang, M. H., Seed-mediated synthesis of high aspect ratio gold nanorods with nitric acid. Chemistry of Materials 2005, 17, (25), 6447-6451.

15. Kim, F.; Sohn, K.; Wu, J. S.; Huang, J. X., Chemical Synthesis of Gold Nanowires in Acidic Solutions. Journal of the American Chemical Society 2008, 130, (44), 14442-+. 
16. Wang, Y.-N.; Wei, W.-T.; Yang, C.-W.; Huang, M. H., Seed-Mediated Growth of Ultralong Gold Nanorods and Nanowires with a Wide Range of Length Tunability. Langmuir 2013, 29, (33), 10491-10497.

17. Lu, X.; Rycenga, M.; Skrabalak, S. E.; Wiley, B.; Xia, Y., Chemical Synthesis of Novel Plasmonic Nanoparticles. Annual Review of Physical Chemistry 2009, 60, (1), 167-192.

18. LaMer, V. K.; Dinegar, R. H., Theory, Production and Mechanism of Formation of Monodispersed Hydrosols. Journal of the American Chemical Society 1950, 72, (11), 4847-4854.

19. Reiss, H., The Growth of Uniform Colloidal Dispersions. The Journal of Chemical Physics 1951, 19, (4), 482-487.

20. Grzelczak, M.; Perez-Juste, J.; Rodriguez-Gonzalez, B.; Spasova, M.; Barsukov, I.; Farle, M.; Liz-Marzan, L. M., Pt-Catalyzed Growth of Ni Nanoparticles in Aqueous CTAB Solution. Chemistry of Materials 2008, 20, (16), 5399-5405.

21. Liu, M. Z.; Guyot-Sionnest, P., Mechanism of silver(I)-assisted growth of gold nanorods and bipyramids. Journal of Physical Chemistry B 2005, 109, (47), 2219222200.

22. Eustis, S.; El-Sayed, M. A., Determination of the aspect ratio statistical distribution of gold nanorods in solution from a theoretical fit of the observed inhomogeneously broadened longitudinal plasmon resonance absorption spectrum. Journal of Applied Physics 2006, 100, (4), 044324-044324-7.

23. Huang, W.-L.; Chen, C.-H.; Huang, M. H., Investigation of the Growth Process of Gold Nanoplates Formed by Thermal Aqueous Solution Approach and the Synthesis of Ultra-Small Gold Nanoplates. The Journal of Physical Chemistry C 2007, 111, (6), 2533-2538.

24. Bastús, N. G.; Comenge, J.; Puntes, V. c., Kinetically Controlled Seeded Growth Synthesis of Citrate-Stabilized Gold Nanoparticles of up to $200 \mathrm{~nm}$ : Size Focusing versus Ostwald Ripening. Langmuir 2011, 27, (17), 11098-11105.

25. Chockla, A. M.; Harris, J. T.; Korgel, B. A., Colloidal Synthesis of Germanium Nanorods. Chemistry of Materials 2011, 23, (7), 1964-1970.

26. Lim, S. I.; Varon, M.; Ojea-Jimenez, I.; Arbiol, J.; Puntes, V., Pt nanocrystal evolution in the presence of $\mathrm{Au}(\mathrm{iii})$-salts at room temperature: spontaneous formation of AuPt heterodimers. J. Mater. Chem. 2011, 21, (31).

27. Johnson, C. J.; Dujardin, E.; Davis, S. A.; Murphy, C. J.; Mann, S., Growth and form of gold nanorods prepared by seed-mediated, surfactant-directed synthesis. Journal of Materials Chemistry 2002, 12, (6), 1765-1770.

28. Nikoobakht, B.; El-Sayed, M. A., Evidence for bilayer assembly of cationic surfactants on the surface of gold nanorods. Langmuir 2001, 17, (20), 6368-6374.

29. Gao, J. X.; Bender, C. M.; Murphy, C. J., Dependence of the gold nanorod aspect ratio on the nature of the directing surfactant in aqueous solution. Langmuir 2003, 19, (21), 9065-9070.

30. Puntes, V. F.; Krishnan, K. M.; Alivisatos, A. P., Colloidal Nanocrystal Shape and Size Control: The Case of Cobalt. Science 2001, 291, (5511), 2115-2117. 
31. Pérez-Juste, J.; Liz-Marzán, L. M.; Carnie, S.; Chan, D. Y. C.; Mulvaney, P., Electric-Field-Directed Growth of Gold Nanorods in Aqueous Surfactant Solutions. Advanced Functional Materials 2004, 14, (6), 571-579.

32. Tornblom, M.; Henriksson, U., Effect of Solubilization of Aliphatic Hydrocarbons on Size and Shape of Rodlike C16TABr Micelles Studied by 2H NMR Relaxation. The Journal of Physical Chemistry B 1997, 101, (31), 6028-6035.

33. Arbiol, J.; Estrade, S.; Prades, J. D.; Cirera, A.; Furtmayr, F.; Stark, C.; Laufer, A.; Stutzmann, M.; Eickhoff, M.; Gass, M. H.; Bleloch, A. L.; Peiro, F.; Morante, J. R., Triple-twin domains in $\mathrm{Mg}$ doped $\mathrm{GaN}$ wurtzite nanowires: structural and electronic properties of this zinc-blende-like stacking. Nanotechnology 2009, 20, (14).

34. Lopez, F. J.; Hemesath, E. R.; Lauhon, L. J., Ordered Stacking Fault Arrays in Silicon Nanowires. Nano Letters 2009, 9, (7), 2774-2779.

35. Arbiol, J.; Comini, E.; Faglia, G.; Sberveglieri, G.; Morante, J. R., Orthorhombic Pbcn SnO2 nanowires for gas sensing applications. Journal of Crystal Growth 2008, 310, (1), 253-260.

36. Arbiol, J.; Morral, A. F. I.; Estrade, S.; Peiro, F.; Kalache, B.; Cabarrocas, P. R. I.; Morante, J. R., Influence of the (111) twinning on the formation of diamond cubic/diamond hexagonal heterostructures in Cu-catalyzed Si nanowires. Journal of Applied Physics 2008, 104, (6).

37. Arbiol, J.; Kalache, B.; Cabarrocas, P. R. I.; Morante, J. R.; Morral, A. F. I., Influence of $\mathrm{Cu}$ as a catalyst on the properties of silicon nanowires synthesized by the vapour-solid-solid mechanism. Nanotechnology 2007, 18, (30).

38. Lim, S. I.; Ojea-Jiménez, I.; Varon, M.; Casals, E.; Arbiol, J.; Puntes, V., Synthesis of Platinum Cubes, Polypods, Cuboctahedrons, and Raspberries Assisted by Cobalt Nanocrystals. Nano Letters 2010, 10, (3), 964-973.

39. Peng, X.; Wickham, J.; Alivisatos, A. P., Kinetics of II-VI and III-V Colloidal Semiconductor Nanocrystal Growth: "Focusing" of Size Distributions. Journal of the American Chemical Society 1998, 120, (21), 5343-5344.

40. González, E.; Arbiol, J.; Puntes, V. F., Carving at the Nanoscale: Sequential Galvanic Exchange and Kirkendall Growth at Room Temperature. Science 2011, 334, (6061), 1377-1380.

41. Kharissova, O. V.; Dias, H. V. R.; Kharisov, B. I.; Perez, B. O.; Perez, V. M. J., The greener synthesis of nanoparticles. Trends in Biotechnology 2013, 31, (4), 240-248.

42. Raveendran, P.; Fu, J.; Wallen, S. L., Completely "green" synthesis and stabilization of metal nanoparticles. Journal of the American Chemical Society 2003, 125, (46), 13940-13941. 